\title{
Screening of Indigenous Yeast From Different Ecological Regions of Kathmandu Valley and Its Application in Wine Production
}

\author{
Bipanab Rajopadhyaya ${ }^{1}$, Bipana Maharjan ${ }^{1}$, Roshani Maharjann ${ }^{1}$, Amrit Acharya ${ }^{1}$ \\ 1 Department Microbiology, Pinnacle College, Affiliated Tribhuvan University, Lalitpur, Nepal
}

Corresponding author: Amrit Acharya, amritnep@gmail.com, ph: 9849180693, Department of Microbiology, Pinnacle College, Affiliated Tribhuvan University, Lalitpur, Nepal University

\begin{abstract}
Objectives: The aim of the study was to isolate and screen the potent yeast from the air for implementing new yeast in wine fermentation.

Methods: In this study, 35 air samples collected in sterile grape juice in glass jar and left over for four days exposure for the growth of yeast from different locations around the Kathmandu Valley. Yeasts were screened by culturing on selective Ethanol Sulfite Agar (ESA) media at $30^{\circ} \mathrm{C}$ for $2-3$ days in Microbiology Lab of Pinnacle College. Yeast isolates were characterized based on colony morphology, microscopic characteristics, Fermentative capacity, Hydrogen sulfide production. Selected yeast isolates were subjected to ethanol fermentation and tested for alcohol tolerance capacity. Wine quality was assessed by sensory evaluation.
\end{abstract}

Results: Of 35 samples, only 20 yeast isolates were isolated. Among these isolates, the variation in colony characteristics along with oval and ellipsoidal microscopic appearance was observed. All the isolates were able to ferment major sugars such as glucose, fructose and sucrose, but few could not ferment galactose and maltose, while none-fermented lactose and xylose. Here, isolates showing no $\mathrm{H}_{2} \mathrm{~S}$ (L29, L34) and mild $\mathrm{H}_{2} \mathrm{~S}$ producer (isolate L31) were subjected to ethanol fermentation. Also, Comparative analysis was made by using commercial standard wine yeast (STAN). Rapid fermentation of grape juice with initial $21^{\circ}$ Brix was observed in L31 isolate, which produced $12.99 \%$ $\mathrm{v} / \mathrm{v}$ alcohol with titratable acidity (TA) $5.25 \mathrm{~g} / \mathrm{L}$, followed by L29 strain with $11.99 \% \mathrm{v} / \mathrm{v}$ alcohol and $4.5 \mathrm{~g} / \mathrm{L}$ TA which were higher than STAN (10.99\% alcohol). These isolates specified as Ethanol tolerance up to $13 \% \mathrm{v} / \mathrm{v}$, while none of them were able to grow at $15 \% \mathrm{v} / \mathrm{v}$ ethanol concentration and $45^{\circ} \mathrm{C}$ temperature. However, significant growth was observed at $\mathrm{pH} 3$ along with sugar tolerance capacity at $30{ }^{\circ} \mathrm{Brix}$. The wine produced by these isolates was found to be remarkably different among each other. While the sensory analysis of wine led to isolate L31 being congenial to tasters.

Conclusion: L31 isolate was found to be efficient and advantageous for wine production indicating its industrial application.

Key words: Yeast, fermentation, colony, Brix, titratable acidity (TA)

\section{INTRODUCTION}

Wine, in the simplest terms, is the fermented grape juice, produced as a result of complex biochemical processes by the synergism of many microbial species normally present on the skin of the grapes, which mainly comprises fungi, yeast and bacteria. Despite the existence of diverse microorganisms on the skin of grapes, only a fraction contributes to the fermentation process in winemaking (Barata et al. 2012). Among

Date of Submission: September 2, 2020

Published Online: December, 2020 which Saccharomyces cerevisiae plays a crucial role in it, converting sugar present in grape juice into alcohol and carbon dioxide, this process is further improved via different non-saccharomyces yeast genera such as Hansenula, Candida, Brettanomyces, Kloeckera, Pichia, Saccharomycodes, Torulopsis (Esteve-Zarzoso et al. 1998) provide significant aroma and taste to wine. Similarly, the most commonly involved bacteria in winemaking are lactic acid bacteria, which are responsible for the

Date of Acceptance: October 7, 2020

DOI: https:/ / doi.org/10.3126/tujm.v7i0.33853 
fermentation of malic acid into lactic acid through a process called malolactic fermentation (Nielsen and Richelieu 1999). Generally, the winemaking process starts through the vinification process to the maceration, where grape juice is extracted from grapes, then it is subjected to the fermentation process from which the wines are transferred into oak barrels for aging. After aging for several months, the wines are fined, filtered and bottled, which is later on supplied to the market (Pretorius 2000). In today's global wine market, a large variety of wines are available that have their own distinct flavors and qualities. These differences among the wines are mostly due to the difference in the variety of grapes used, viticulture practices, art of winemaker and the use of innovative technology (Bisson 2002). However, primarily it depends upon the type of Saccharomyces yeast strain used, as different strains of the yeast provide a different flavor to the wine and gives uniqueness to the product (Romano et al. 2003). Over the years, much research has been conducted to isolate various wild strains of Saccharomyces species for improving ongoing wine fermentation and to obtain the best wine out of it. Nowadays, hundreds of different strains of Saccharomyces have been commercialized for winemaking, which is called wine yeast. This commercialization of wine yeast is mostly done by selecting the isolate possessing these characteristics, such as quick initiation of fermentation, tolerance to low $\mathrm{p}^{\mathrm{H}}$, high temperature, high sugar and ethanol concentration, low production of hydrogen sulfide, low requirement of nitrogen, production of desirable bouquet and ability to ferment up to dryness (Rainieri and Pretorius 2000). Today, most of the wild strains are genetically modified to enhance their overall characteristics and performance. In spite of the availability of such wine yeast for industrial fermentation, there is still a need of improvement in the existing ones because the potent wine yeast strain has been rarely isolated and exploration of the natural diversity of these yeasts has not yet been accomplished (Wang et al. 2012).This research isolates such indigenous species of Saccharomyces present in Kathmandu Valley as it bears different climatic and geographical differences within a small area. It comprises a subtropical to temperate rainforest with a pleasant climate warm in days followed by cool morning and night. The temperature during summer varies from $28-30^{\circ} \mathrm{C}$ while in winter around $3-10^{\circ} \mathrm{C}$ and the humidity is approximately $75 \%$ with rainfall total monsoon based. The valley is surrounded by four hills; Phulchowki in South-East, Chandragiri in South-West, Shivapuri in North-West and Nagarkot in the NorthEast with altitudes ranging from 2000- $2831 \mathrm{~m}$ (Jha 2016) and the yeast adapted within this region could be different with different fermentative capacity (Spencer 2013).In this study, isolation and characterization of wild-type strains of Saccharomyces have been discussed and its uses in winemaking have been interpreted. Hence, this study aids in selecting and improve the wine quality along with its implementation of potent wild yeast isolates of Saccharomyces, which will create a strong identity that will eventually facilitate its application in winemaking.

\section{MATERIALS AND METHODS}

Sample size, site and sample collection: A total of 35 air samples were examined randomly from different places of Kathmandu valley (12 samples collected from Katmandu, Bhaktapur (11), and Lalitpur (12) respectively).

For sampling, the grape juice of $100 \mathrm{ml}$ approx. was poured in heat resistant and leak proof glass jar of $400 \mathrm{~mL}$ and the muslin cloth (15x17-cm length and breadth) wrapped in aluminum foil (to prevent moistening during sterilization) was autoclaved. After sterilization, the glass jar and the muslin cloth were placed into sterile plastic bags before shifting it to the sampling site. After transporting to sampling sites, the lid of the jar was exposed while its mouth was covered with muslin cloth and was left over for four days as an exposure period for the growth of yeast in the sampling site, which was collected and labeled with specific codes

Isolation and identification of Saccharomyces: For isolating Saccharomyces, collected samples were further incubated at $30^{\circ} \mathrm{C}$ for approximately one week in the lab, from which a loop full of a suspension was streaked onto the selective medium i.e. Ethanol Sulfite Agar (ESA), which was then incubated at $30^{\circ} \mathrm{C}$ for $2-3$ days. The growth of colonies was then sub cultured on the Yeast Extract Peptone D-glucose Agar (YEPDA) for further identification (Kish et al. 1983).

Culture characteristics and microscopic identification: Cultural characteristics such as shape, size, color, margin, surface, elevation, consistency and opacity of the single isolated colony were noted along with microscopic identification was performed using a 
simple staining method

Fermentative capacity testing: Yeast fermentation broth (YFB) containing inverted Durham's tube was used to identify Saccharomyces species based on the fermentation of different carbohydrate sources (Glucose, Fructose, Sucrose, Galactose, Lactose, Maltose and Xylose). The YFB media were prepared by the addition of peptone $7.5 \mathrm{~g}$, yeast extract $4.5 \mathrm{~g}, 1 \mathrm{ml}$ of $1.6 \mathrm{w} / \mathrm{v}$ bromothymol blue as an indicator into 1000 $\mathrm{ml}$ distilled water which was boiled then $7 \mathrm{~mL}$ of each solution was poured into each test tubes along with Durham's tubes, whereas $5 \% \mathrm{w} / \mathrm{v}$ of each carbohydrate solution was prepared separately in $3 \mathrm{ml}$ distilled water and was autoclaved. After sterilization, the media was allowed to cool at $40-45^{\circ} \mathrm{C}$ then the different carbohydrate solution was poured into respective tubes along with loop full of suspension of isolate into each medium and incubated at $30^{\circ} \mathrm{C}$ for 3 days and the result was indicated by change in the color of media from green to either yellow (if acidic) or blue (if alkaline) and the presence of gas in the Durham's tube along with control tubes in each set to monitor the contamination of the medium (Guimaraes et al. 2006).

Hydrogen sulfide testing: $\mathrm{H} 2 \mathrm{~S}$ production test of the isolates was performed by growing yeast isolates on Lead Acetate Agar (LAA) medium (40 g/l glucose, $5 \mathrm{~g} / 1$ yeast extract, $3 \mathrm{~g} / 1$ peptone, $0.2 \mathrm{~g} / 1$ ammonium sulfate, $1 \mathrm{~g} / 1$ lead acetate, $20 \mathrm{~g} / 1$ agar) and the plates were incubated at $30^{\circ} \mathrm{C}$ for 10 days. The result was indicated positive by observing dark brown colonies of isolates on lead acetate medium and vice versa (Ono et al. 1991).

Alcohol fermentation: The isolates producing less or no H2S gas were subjected to the alcohol fermentation which was performed in the glass bottles of $450 \mathrm{ml}$ and $400 \mathrm{ml}$ of freshly pressed grape juice was poured with the sugar concentration around $20^{\circ}$ Brix along with initial $\mathrm{pH}$ reading of grape juice, which was then autoclave for sterilization, then the suspension of selected isolates equivalent to 2 McFarland was poured and was fermented at $25^{\circ} \mathrm{C}$. The reduced sugar concentration was monitored for weeks with one-day gap intervals until concurrent Brix reading was observed. The amount of Ethanol produced was measured by comparing the specific gravity reading of the distillate to the standard chart and the result was interpreted as $\% \mathrm{v} / \mathrm{v}$ (percentage volume by volume).
Also, the final $\mathrm{PH}$ and the titratable acidity of the grape must be measured (Shrestha et al. 2002; Jacobson 2006).

Stress tolerance test: To determine the tolerance capacity of selected isolates at different alcohol concentration and sugar concentrations followed by temperature and $\mathrm{PH}$. The isolates were first grown in YEPD medium with slight modification where sucrose was used instead of glucose and its concentration was maintained at $200 \mathrm{~g} / 1$ (20 nb ${ }^{\circ}$ Brix) and the PH was maintained around 3.5 and was supplemented with 80 $\mathrm{ml} /$ liter of ethanol and incubated at $30^{\circ} \mathrm{C}$ for 3 days. The isolates from this medium were then subjected to Yeast Extract Peptone Broth (YEPB) supplemented with $10 \mathrm{~g} / 1$ yeast extract, $20 \mathrm{~g} / 1$ peptone for respective tolerance test the concentration of sucrose was chosen as required (Guimaraes et al. 2006).

Alcohol tolerance test: The isolates were inoculated into (YEP) broth supplemented with $100 \mathrm{ml} / 1,130$ $\mathrm{ml} \backslash 1,150 \mathrm{ml} / 1$ ethanol solution respectively was then incubated at $30^{\circ} \mathrm{C}$ for 3 days and the growth was monitored by measuring the optical density at $540 \mathrm{~nm}$ with the use of a colorimeter.

Temperature tolerance test: The isolates were inoculated into (YEP) broth, which were incubated in $5^{\circ} \mathrm{C}, 25^{\circ} \mathrm{C}, 35^{\circ} \mathrm{C}$, and $45^{\circ} \mathrm{C}$ respectively for 3 days and the growth was monitored by measuring the optical density at $540 \mathrm{~nm}$ with the use of a colorimeter

pH tolerance test: The isolates were inoculated into (YEP) broth, where its $\mathrm{PH}$ is maintained at 3, 3.5, 4, and 4.5 , respectively and incubated at $30^{\circ} \mathrm{C}$ for 3 days and the growth was monitored by measuring the optical density at $540 \mathrm{~nm}$ with the use of colorimetry.

Sugar tolerance test: The isolates were inoculated into YEP broth in which sugar concentration was maintained at $10^{\circ}, 20^{\circ}, 30^{\circ}, 40^{\circ}$, and $50^{\circ}$ Brix and it was incubated at $30^{\circ} \mathrm{C}$ for 3 days and the growth was monitored by measuring the optical density at $540 \mathrm{~nm}$ with the use of a colorimeter.

Sensory analysis of wine: In sensory analysis of selected isolates, a freshly pressed grape juice of approximately $500 \mathrm{ml}$ was poured into a glass bottle of $750 \mathrm{ml}$ with sugar concentration was maintained around $24^{\circ}$ Brix and initial $\mathrm{PH}$ 4.6. The must was supplemented with $50 \mathrm{mg} / 1$ potassium metabisulphite and $100 \mathrm{mg} / 1$ Ammonium sulfate then suspension of the selected isolates equivalent to 2 McFarland 
was poured into each glass bottle and was allowed to ferment at $25^{\circ} \mathrm{C}$ for 1 month. After a month of fermentation and removing sediments, the clarified wines were subjected to evaluation by 5 experienced tasters including wine quality manager. The sensory attributes (appearance, odor, taste and body of a wine) were used to estimate the quality among the tasters, and the divergence was evaluated by the score given by five tasters on each category of sensory attributes using two-way ANOVA table (for repeated values) along with rating based on their perception from 1 to 5 where $1=$ Not bad, 2= Satisfactory, $3=$ good, $4=$ better and $5=$ excellent (Jackson $2008 \& 2009$ ).

Quality control: Standard procedure and strict aseptic conditions were maintained to obtain reliable results and sterility of each media from a batch was checked by incubating one media of each batch in an incubator at $37^{\circ} \mathrm{C}$ for $24 \mathrm{hrs}$. For comparative analysis, the standard wine producing strain Saccharomyces cerevisiae (springer Oenologie, fermentis, Belgium) culture was used.

\section{RESULTS}

Out of 35 air samples, 11(31.42\%) from Bhaktapur, $12(34.29 \%)$ from Lalitpur and $12(34.29 \%)$ from Kathmandu district were examined. The significant growth was observed in $66.67 \%(8 / 12)$ of Kathmandu samples. The culture was positive for $58.33 \%(7 / 12)$ and $45.45 \%$ (5/11) of Lalitpur samples and Bhaktapur samples respectively. On selective ESA media, only 20 samples indicated growth as yeast. These yeast isolates on YEPD agar medium exhibited circular opaque and mucoid characteristics along with umbonate elevation and entire margin, similarly white colony was observed as shown in Fig-1A, which we found similar to the colony characteristics of the standard commercial yeast used in the study. The colony on YEPD agar medium of selected isolates is shown in the figure:2A. Microscopic characteristics of the isolates revealed an oval and ellipsoidal appearance, which resembles the characteristics of Saccharomyces species.

All the isolates were able to ferment major sugar, such as glucose, fructose and sucrose, whereas some isolates were unable to ferment maltose and galactose and none of them were able to ferment xylose and lactose as shown in Fig-1B. This result also signifies variance in sugar assimilation properties among the Saccharomyces species, which further aid in identification as Saccharomyces species as isolates were unable to ferment lactose and xylose sugar.

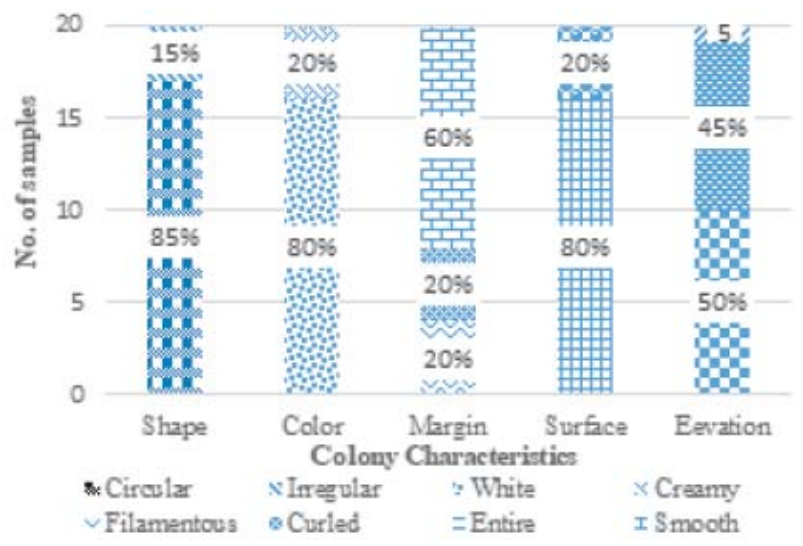

(A)

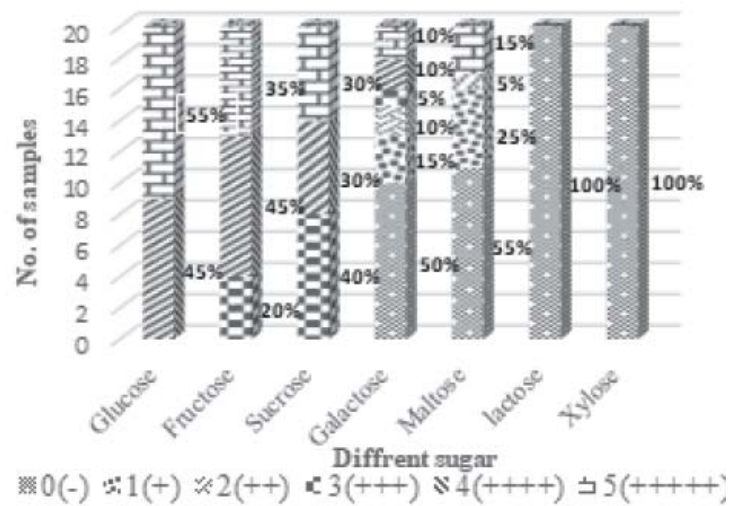

(B)

Figure 1: (A) Colony characteristics of the yeast isolates and (B) Fermentative capacity of different sugars by yeast isolates 


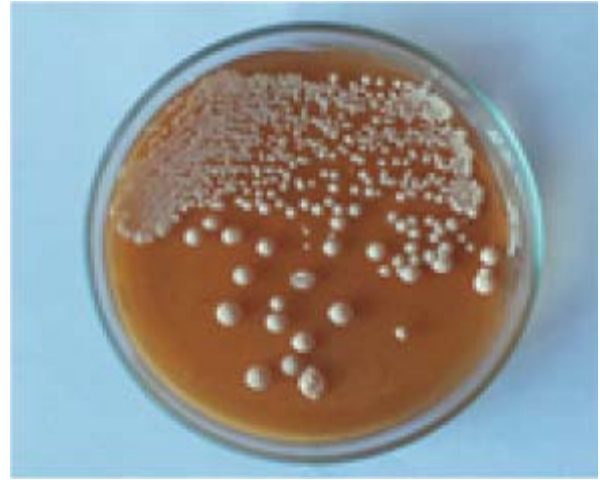

(A)

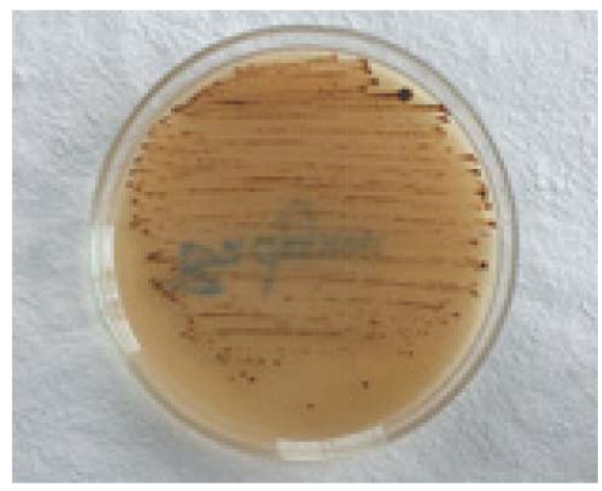

(C)

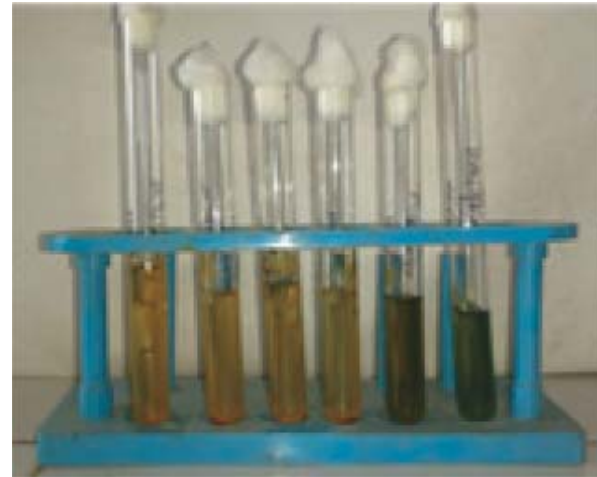

(B)

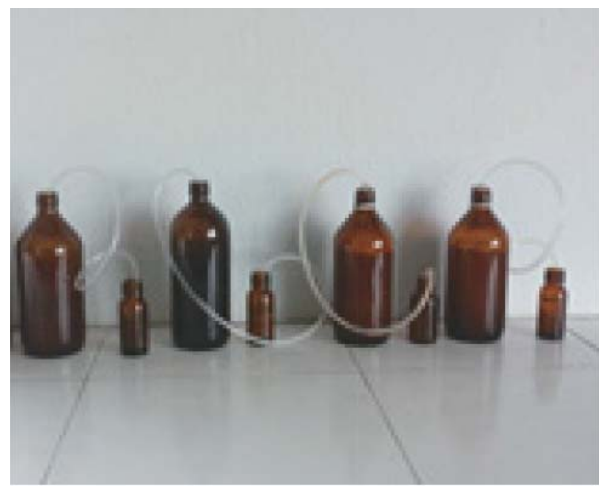

(D)

Figure 2: (A) Colony characteristics (B) Fermentative Characteristics (C) Dark black colonies on Lead Acetate Agar (D) Ethanol fermentation

In this study, we found that $2(15 \%)$ samples were found to be hydrogen sulfide non-producer i.e. absence, 1 (5\%) samples was found to be a mild hydrogen sulfide producer, 8 (35\%) samples were found to be moderate hydrogen sulfide producer and 9 (45\%) samples were found to be intense hydrogen sulfide producer as shown in Fig-3. Since, H2S can cause rotten egg aroma in the wine, hence only $\mathrm{H}_{2} \mathrm{~S}$ negative and mild $\mathrm{H}_{2} \mathrm{~S}$ positive isolates were chosen for further study parameters.

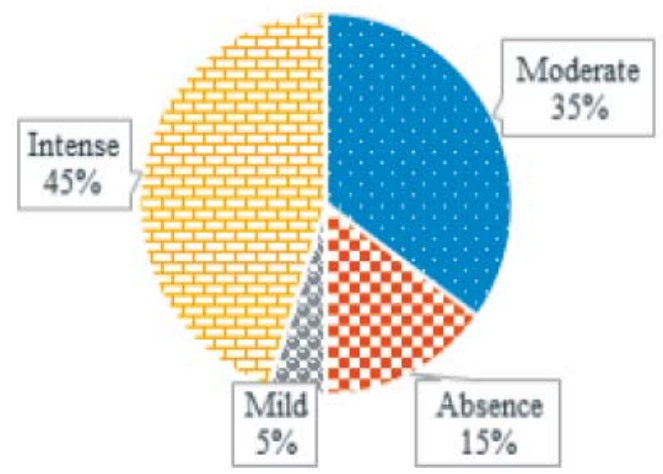

Figure 3: Comparison of Hydrogen sulfide production

\section{Ethanol Fermentation}

Isolates showing no H2S (L29, L34) and mild H2S production (L31) were subjected to ethanol fermentation, where grape juice with initial $21{ }^{0}$ Brix was taken as a substrate, and isolate with 2 Mcfarland turbidity was inoculated into the fermentation vessel, and sugar assimilation were noted on alternate day. In this study we observe that, the significant decrease 
of $21^{\circ}$ Brix within a 5 th day was 9 shown by L29 and STAN, while least was found to be 16 as shown by L31. After the 7 th day, ${ }^{0}$ Brix was 7 for L29 \& 6.5 for L31 but for STAN's ${ }^{0}$ Brix was 6 . Since L34 expressed stuck and sluggish fermentation as shown in Fig-4A So, it was excluded from further processing. Similarly, ethanol production was found to be $12.99 \%$ by L31 followed by $11.99 \%$ L29 and the least by $10.99 \%$ STAN. However, the titratable acidity of grape must be found to be highest by $5.25 \mathrm{~g} / \mathrm{L}$ of L31 followed by $4.5 \mathrm{~g} / \mathrm{L}$ of STAN and the least was found to be $4.05 \mathrm{~g} / \mathrm{L}$.

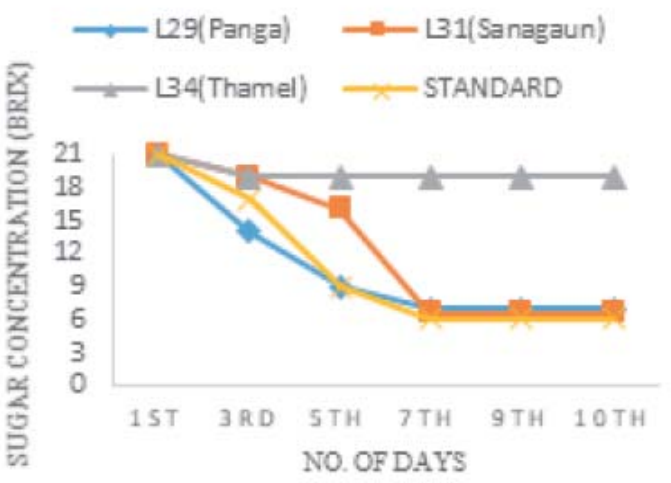

(A)

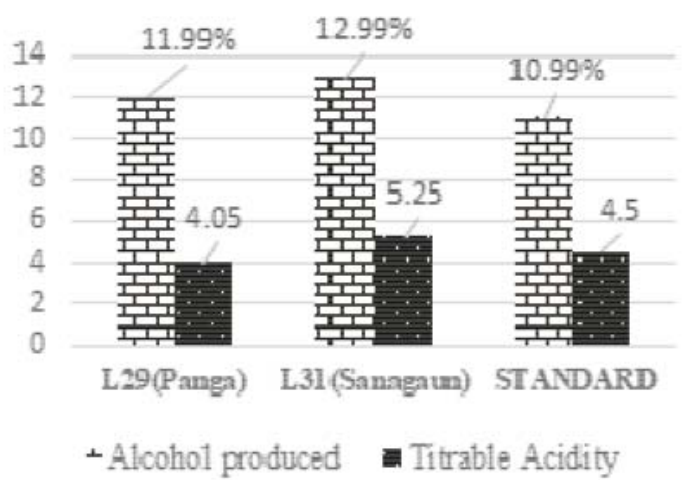

(B)

Figure 4: (A) Sugar utilization of selected isolates and standard (B) Comparison of alcohol produced and titratable acidity of selected yeast and standard

\section{Stress tolerant test}

The temperature tolerance test: The temperature tolerance capacity of the selected yeast showed notable variation. The cardinal temperature of temperature tolerance is at $5^{\circ} \mathrm{C} \& 45^{\circ} \mathrm{C}$ (minimum), $25^{\circ} \mathrm{C}$ (maximum) and $35^{\circ} \mathrm{C}$ (optimal). Despite some fluctuation, there was a gradual increment of tolerance capacity leading by L31, which peaked at $25^{\circ} \mathrm{C}$ by 1.91 (O.D.) followed by L291.91 (O.D.) and Standard by 1.67 (O.D.) respectively as shown in the Fig-5A.. After $25^{\circ} \mathrm{C}$, there was a rapid decrease in temperature tolerance capacity of the selected strains, including standard, which dropped to null at $45^{\circ} \mathrm{C}$ by all yeast isolates.

Sugar tolerance test: There was a significant increment of sugar tolerance capacity from 10 to $20^{\circ}$ Brix in which L29 showed maximum viable growth of 0.91 (O.D.) followed by 1.86 (O.D.) and 1.76 (O.D.) of L31 and Standard, respectively, as shown in the Fig-5B.. After 20 Brix sugar concentration, there was a sharp decrement of viable growth, which eventually stopped at 40 and 50 Brix with no tolerance capacity from the selected strains along with standard.
Alcohol tolerance test: The alcohol tolerance capacity of the selected yeast showed notable variation by the gradual decrease in the difference in viable count by the colorimeter (O.D.) with increment of the alcohol concentration. L31 yeast isolate was leading among selected yeast isolates throughout all alcohol concentration followed by L29 and Standard. The maximum tolerance capacity can be observed at $10 \%$ alcohol concentration by 0.17 (O.D.) L31, followed by L29 0.10 (O.D) and by Standard 0.09 (O.D), which decreased at $13 \%$ alcohol concentration to 0.07 (O.D) of L31 and 0.04 (O.D.) of L29 and Standard by 0.02 (O.D). In 15\% alcoholic concentration, no growth was observed, L31 showed the tolerance by 0 (O.D) followed by L29 by 0 (O.D) and Standard by 0 (O.D) as shown in the Fig-5(C).

pH tolerance test: The maximum tolerance capacity and growth of viability was shown by L29 from 0.16 to $1.2(\mathrm{O} . \mathrm{D})$ as the $\mathrm{P}^{\mathrm{H}}$ concentration increased from 3 to 4.5 followed by L31 from 0.13 to 1.19 (O.D). The least tolerance and growth were shown by Standard from 0.13 to $1.07(\mathrm{O} . \mathrm{D})$ as the $\mathrm{P}^{\mathrm{H}}$ concentration increased from 3 to 4.5, as shown in the Fig-5D. 


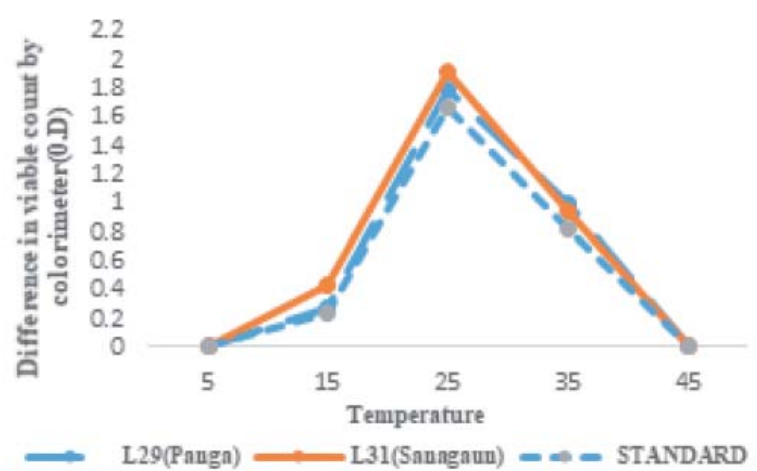

(A)

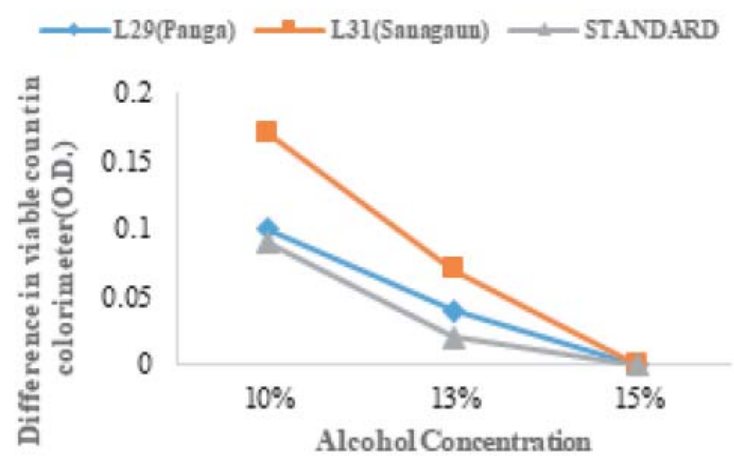

(C)

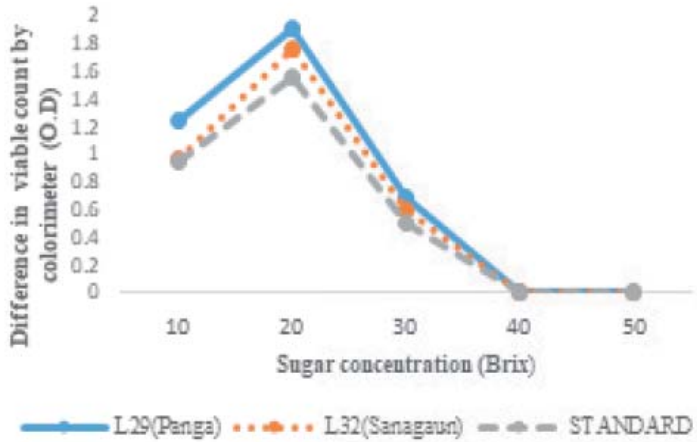

(B)

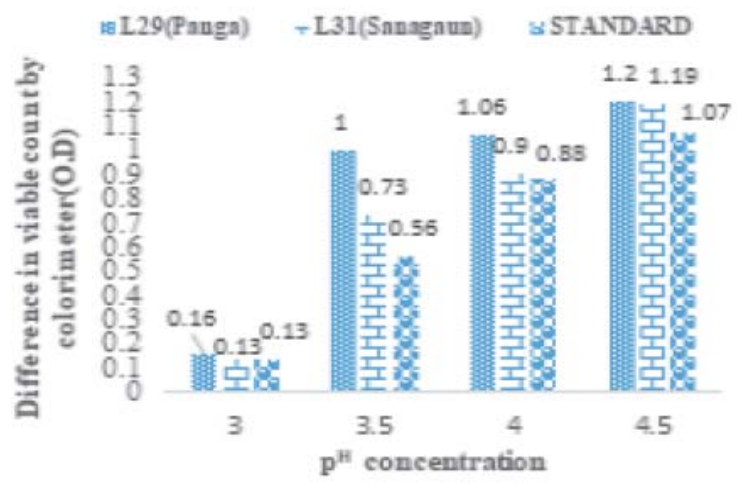

(D)

Figure 5: (A) Temperature Tolerance Capacity (B) Sugar Tolerance Capacity (C) Alcohol Tolerance Capacity and (D) pH Tolerance Capacity

Sensory analysis of wine: Sensory analysis was reported for three wines by five testers. According to them, the variance among the wines in their appearance, odor, taste and body was analyzed in which the scoring was manifested that there was a significant difference between the wines and tasters as the value of F-calculated exceeded that of F-tabular at $5 \%$ level of significance.

Table : Two-way ANOVA table designed for repeated value

\begin{tabular}{lccccccc}
\hline \multicolumn{1}{c}{ Source } & $\mathrm{SS}$ & $\mathrm{d}$.f. & $\mathrm{m} . \mathrm{s}$ & $\mathrm{F}$ & $\mathrm{F}_{0.10}$ & $\mathrm{~F}_{0.05}$ & $\mathrm{~F}_{0.01}$ \\
\hline Total & 152 & 15 & & & & & \\
Wines & 8.4 & 4 & 2.1 & 5.1686 & 2.8064 & $3.84^{* *}$ & 7.006 \\
Tasters & 77.9999 & 2 & 38.9995 & 95.9869 & 3.1131 & $4.46^{* *}$ & 8.649 \\
Interaction & 62.3501 & $1^{*}$ & 62.3501 & 153.4583 & 3.4579 & $5.32^{* *}$ & 11.259 \\
Error & 3.25 & 8 & 0.4063 & & & & \\
\hline
\end{tabular}

** Significance at $5 \%$ level(SS =Type III sum of square, $m . s=$ Mean square, $d . f=$ degree of freedom, $\mathrm{F}=$ Test statistic $F$ )

Rating of wine by five testers: The selected isolated strains were processed for fermentation of grape must for the preparation of wine and named them wine A, B, and C for strains Standard, L31 and L29, respectively. From the sensory analysis conducted among five tasters, 2 from wine A, 3 from wine B and 1 from wine
$C$ rated satisfactory as a result, while remaining 3 from wine A, 2 from wine $B$ and 3 from wine $C$ rated good, while 1 taster rated better for wine $C$. Hence, wine $C$ was found to be comparatively better than wine $B$ and wine $\mathrm{C}$ as shown in Fig-6. 


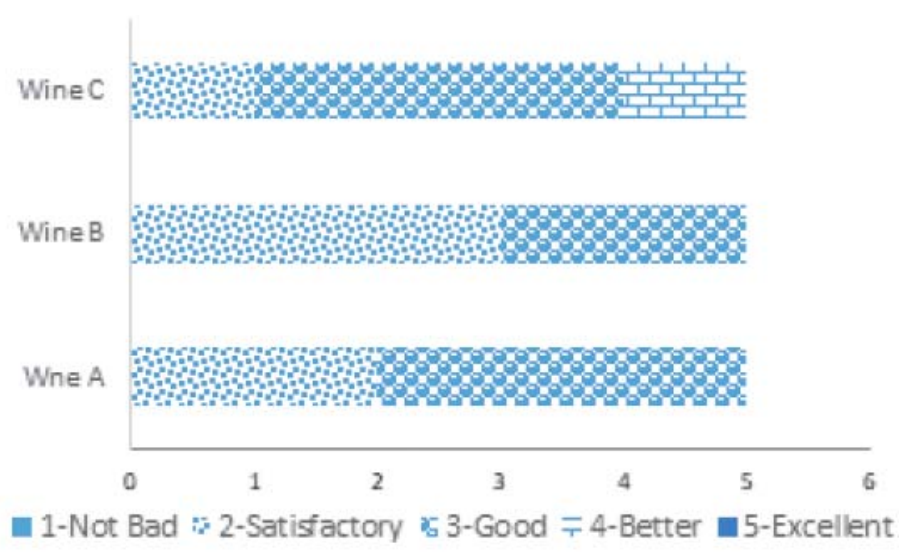

Figure 6: Comparison of the ratings for three wines by five tasters

\section{DISCUSSION}

Of 35 samples were examined, only 20 indigenous Saccharomyces species were isolated. Ethanol sulfite agar media were used to isolate selectively for yeast, where 1 from Bhaktapur, 9 from Lalitpur and 10 from Kathmandu was isolated, which may be due to its low occurrence in nature. A similar result was observed by (Ghosh et al. 2013) in which out of 26 ascomycetous isolates only 2 yeast isolates were discovered to be Saccharomyces species. Additionally (Martini et al. 1996) has reported the rare isolation of Saccharomyces from the grape skin, which was noted to be only 3 isolates among 30 samples.

Colony characteristics (shape, surface, margin and elevation) of the 20 isolates were observed, which expressed similar colony characteristics with standard commercial yeast (STAN). Their colony characteristics were noticed to be circular, smooth and creamy color colonies along with umbonate elevation with an entire margin and were opaque. Similar findings were also observed by (Asyikeen et al. 2013), in which 18 of the yeast isolates were opaque, smooth, regular colonies and creamy in color but other 2 yeast isolates were similar except rough colonies were observed. A rough colony formed by wild Saccharomyces has also been reported by (Kuthan et al. 2003).

In this study, simple staining was performed for microscopic identification of isolates, which demonstrated ellipsoidal or oval-shaped proving the characteristics of Saccharomyces species. Similarly, the fermentative characteristics of 20 isolates expressed variant sugar assimilation profile as all isolates could ferment major sugar, such as glucose, sucrose and fructose, while most of the strains were unable to ferment galactose and maltose and none of the isolates were able to ferment xylose and lactose. From the sugar assimilation profile, the yeast isolates were further identified as Saccharomyces species as none of the isolates assimilated lactose and xylose (Guimaraes et al. 2006). Also, (El-Nemr 2001) reported that lactose sugar was unable to be fermented due to the absence of a b-galactosidase enzyme among the Saccharomyces species.

This study observed that among the total 20 yeast isolates, $2(15 \%)$ samples were noted to be hydrogen sulfide non-producer i.e. absence, while rest $18(85 \%)$ isolates were hydrogen sulfide producer. Among them, 1 (5\%) samples were mild hydrogen sulfide producer, $8(35 \%)$ samples were moderate hydrogen sulfide producers and $9(45 \%)$ samples were intense hydrogen sulfide producer, where the proportion of non- hydrogen sulfide producing wine yeast was rarely present in nature as isolates L29 and L34 implies non-hydrogen sulfide producers. A similar result was discovered by (Asyikeen et al. 2013) where among 16 tentative wild yeast isolates, only 2 isolates were non- hydrogen sulfide producers. Ugliano et al. (2011) reported that the isolate with characteristics of hydrogen sulfide was of no use for wine fermentation as it imparts the off odors described as rotten egg and/ or sewage to wine. So, the isolates with a high profile of producing Hydrogen sulfide i.e. (moderate and intense) were excluded from the study.

During the study, on subjecting the H2S negative isolate L29, L34 and Low H2S isolate L31 to alcohol fermentation discovered significant decrement of 21 Brix within a 5th day, which was 9 shown by L29 and STAN, while least was found to be 16 by isolate 
L31. After the 7th day, Brix was noted to be 7 by L29 \& 6.5 by L31 but for STAN, Brix was observed to be 6. However, the isolates L34 noted no decrement of sugar by 18 Brix within a 7th day. Therefore, isolate L34 was excluded from further processing, as stuck and sluggish fermentation processes were found to be problematic for wine production (Bisson 1999).

In recent studies, the maximum alcohol produced via specific gravity was noted to be $12.99 \%$ by L31 followed by L29 (11.99\%) and the least by STAN (10.99\%). However, the titratable acidity of grape must was found to be highest in L31 $(5.25 \mathrm{~g} / \mathrm{L})$ followed by STAN $(4.5 \mathrm{~g} / \mathrm{L})$ and the least was observed to be $4.05 \mathrm{~g} / \mathrm{L}$ by L29. A similar study was reported by (Callejon et al. 2010) in which the final alcohol concentration of four wild yeast isolates ranges from 11.9 to $12.6 \% \mathrm{v} / \mathrm{v}$.

In this study, the temperature tolerance capacity of the selected yeast isolates expressed notable variation. The cardinal temperature of temperature tolerance is at $5^{\circ} \mathrm{C} \& 45^{\circ} \mathrm{C}$ (minimum), $25^{\circ} \mathrm{C}$ (maximum) and $35^{\circ} \mathrm{C}$ (optimal). Despite some fluctuation, there was a gradual increment of tolerance capacity leading by L31, which peaked at $25^{\circ} \mathrm{C}$ by 1.91 (OD) followed by L29 1.91 (OD) and STAN by 1.67 (OD), respectively. After $25^{\circ} \mathrm{C}$, rapid decrement of temperature tolerance capacity by all selected isolates including STAN dropped to null reaching at $45^{\circ} \mathrm{C}$. This result signifies that the appropriate temperature-maintained leads to increased growth, metabolism and survival of yeast during fermentation. A similar result was obtained by (Asyikeen et al. 2013), in which wild yeast isolated from various fruits and other plant materials could not grow at the high temperature of $37^{\circ} \mathrm{C}$. (Torija et al. 2003) reported that fermentation of different strains in white must was dynamic at high temperature between intervals of $30-35^{\circ} \mathrm{C}$. Additionally, (Bertolini et al. 1991) has reported no growth due to the effect of high temperature on $40^{\circ} \mathrm{C}$.

In this research conducted, there was a significant increment of sugar tolerance capacity of selected isolates from 10 to 20 Brix in which significant growth of 0.91 (OD) followed by 1.86 (OD) and 1.76 (OD) of L29, L31, and STAN, respectively. After 20 Brix sugar concentration, there was a sharp decrement of viable growth, which eventually stopped at 40 and 50 Brix with no tolerance capacity from the selected strains along with STAN. Hence, all the isolates showed sugar tolerance capacity at 30 Brix. This result indicated that higher the sugar concentration, greater the inhibitory effect due to osmotic stress. A similar result was reported (Bertolini et al. 1991) that the strains they isolated from Brazilian alcohol factories were potential enough to ferment up to $30 \%$ of sucrose readily. However, the sugar concentration of $200 \mathrm{~g} / \mathrm{L}$ to $300 \mathrm{~g} / \mathrm{L}$ decreased Saccharomyces cerevisiae growth rate as reported by (Charoenchai et al. 1998). Which signifies that the greater the sugar concentration greater the inhibitory effect on the yeast cell.

This study showed that the alcohol tolerance capacity of the selected yeast isolates revealed notable variation by the gradual decrement in a difference in the viable count by the colorimeter (OD) with an increment of the alcohol concentration. L31 yeast isolate was leading among selected yeast isolates throughout all alcohol concentration followed by L29 and STAN. The research conducted showed that the maximum tolerance capacity was observed at $10 \%$ alcohol concentration by L31 0.17 (OD), followed by L29 0.10 (OD) and by STAN 0.09 (OD), which decreased at $13 \%$ alcohol concentration to 0.08 (OD) of L31 and 0.04 (OD) of L29 and STAN by 0.02 (OD). In 15\% alcoholic concentration, all the yeast isolates, including standard manifested no visible growth i.e. 0 (OD). This indicates that higher ethanol concentrations minimum the growth of yeast cells, which unfortunately cease the fermentation process. Similarly, higher ethanol produced during fermentation leads to inhibitory effect was also reported by (Wayman and Parekh 1990) In contrast, most of the ethanol-resistant yeast isolation has been reported by (Umeh 2016). While in this study selected yeast isolates along with standard commercial yeast showed no ethanol tolerance above $13 \% \mathrm{v} / \mathrm{v}$. The study noticed that the maximum tolerance capacity and growth of viability was shown by L29 from 0.16 to 1.2 as the $\mathrm{pH}$ concentration increased from 3 to 4.5 followed by L31 from 0.13 to 1.19 . The least tolerance and growth were shown by STAN from 0.13 to 1.07 as the $\mathrm{pH}$ concentration increased from 3 to 4.5 . This study implies that the best $\mathrm{pH}$ tolerance capacity due to the tolerance of $\mathrm{H}+$ ions excreted by yeast and eventually drops of the $\mathrm{pH}$ of the media. A similar report was reported by (Charoenchai et al. 1998) reported that the $\mathrm{pH}$ range between 3 and 4 does not affect the growth rate and cell biomass of wine yeast. This indicates that yeast isolates in this study showed tolerance capacity at 
$\mathrm{pH}$ concentration 3 .

In this study, the wine produced by the selected isolates was noted to be significantly different from each other, which signifies that the flavor profile of the particular wine is influenced by the different types of yeast used (Romano et al. 2008). In addition, also by analyzing the ratings given to particular wine by five experienced tasters revealed that the highest vote was manifested to be in the wine produced by L31 (i.e. 3 tasters voted for good, 1 voted for the satisfactory and 1 voted for better) which is then followed by STAN (i.e. 3 tasters voted for good and 2 voted for satisfactory) While in the wine produce by L29 3 tasters voted for satisfactory and 2 voted for good. This result signifies that the wine produced by L31 was found to be mostly likable among the tasters.

\section{CONCLUSION}

Indigenous yeast isolated from the air samples in Kathmandu were more potential than a standard industrial yeast when compared the physiological characteristics including growth profile, appearance, fermentative capacity, hydrogen sulfide, tolerance capacity (such as temperature, sugar, alcohol and $\mathrm{pH}$ ), alcoholic fermentation and sensory analysis. All yeast isolates fermented sugars such as glucose, sucrose and fructose, but only a few fermented galactose and maltose and none-fermented lactose and xylose sugars enabling yeast identification. From the total yeast isolates, only 2 (15\%) yeasts are hydrogen sulfide non-producer, which is lowly compared to the H2S producer i.e. $18(85 \%)$. The selected yeast isolates are capable of producing high ethanol concentration with significant titratable acidity compared to commercial yeast which i.e. $11.99 \%$ and $4.5 \mathrm{~g} / \mathrm{L}$ for $\mathrm{L} 29,12.99 \%$, and $5.25 \mathrm{~g} / \mathrm{L}$ for L31 and 10.99\%, and $4.05 \mathrm{~g} / \mathrm{L}$ for (STAN) commercial yeast, respectively. These isolates were able to tolerate up to $13 \%$ ethanol concentration along with visible growth at $\mathrm{pH} 3$ and sugar concentration of 30 Brix with no thermotolerant capacity at $45^{\circ} \mathrm{C}$. Similarly, the sensory analysis revealed the diversity of wine produced and wine produced by the isolate L31 seems most likable among the tasters. Hence, it signifies that the yeast isolates L29 and L31 are superior to commercial strain, which enables them for industrial application.

\section{ACKNOWLEDGEMENT}

We would like to express our sincere gratitude and appreciation to all the faculty members Mr. Umesh Manandhar, Mr. Biraj Lohani and Mr. Krishna Sushil Shrestha, Mr. Dipendra Yadhav of the Pinnacle College for their generous advice and support as well as guidance to complete this study.

\section{CONFLICT OF INTEREST}

The authors declare that they have no competing interests

\section{REFERENCES}

Asyikeen ZN, Maaruf A, Sahilah A, Khan AM and Aida WW (2013) A new source of Saccharomyces cerevisiae as a leavening agent in bread making. Int Food Res J 20(2).

Barata A, Malfeito-Ferreira M and Loureiro V (2012) The microbial ecology of wine grape berries. Int J Food Microb 153(3): 243-259.

Bertolini MC, Ernandes J and Laluce C (1991) New yeast strains for alcoholic fermentation at higher sugar concentration. Biotechnol lett 13(3): 197-202.

Bisson LF (1999) Stuck and sluggish fermentations. Am J Enol Vitic 50(1):107-119.

Bisson LF, Waterhouse AL, Ebeler SE, Walker MA and Lapsley JT (2002) The present and future of the international wine industry. Nature 418(6898): 696.

Callejon R, Clavijo A, Ortigueira P, Troncoso A, Paneque P and Morales M (2010) Volatile and sensory profile of organic red wines produced by different selected autochthonous and commercial Saccharomyces cerevisiae strains. Analytica Chimica Acta 660(1): 68-75.

Charoenchai C, Fleet GH and Henschke PA (1998). Effects of temperature, $\mathrm{pH}$, and sugar concentration on the growth rates and cell biomass of wine yeasts. Am J Enol Vitic 49(3): 283288.

El-Nemr TM (2001) Immobilization of recombinant strains of Saccharomyces cerevisiae for the hydrolysis of lactose in salted Domiati cheese whey. Eur Food Res Technol 212(2): 225-227.

Esteve-Zarzoso B, Manzanares P, Ramon D and Querol A (1998) The role of non-Saccharomyces yeasts in industrial winemaking. Int Microbiol 1(2): 143-148.

Ghosh S, Santra T and Chakravarty A (2013) Study of 
antagonistic yeasts isolated from some natural sources of West Bengal. Agri Biol JN Am 4: 33-40.

Guimarães TM, Moriel DG, Machado IP, Picheth CM and Bonfim $\mathrm{T}$ (2006) Isolation and characterization of Saccharomyces cerevisiae strains of winery interest. Braz J Pharm Sci 42(1): 119-126.

Jackson RS (2009) Wine Tasting: A Professional Handbook: Elsevier Science. 2nd edition pp 226242

Jacobson JL (2006) Introduction to Wine Laboratory Practices and Procedures: Springer US.

Jha SK (2016). Studies on macrofungal diversity of Kathmandu Valley, Nepal and nutritive value of some dominant edible ones.

Kish S, Sharf R, and Margalith P (1983) A note on a selective medium for wine yeasts. J Appl Microbiol 55 (1): 177-179.

Kuthan M, Devaux F, Janderova B, Slaninova I, Jacq C and Palkova Z (2003) Domestication of wild Saccharomyces cerevisiae is accompanied by changes in gene expression and colony morphology. Mol microb 47(3): 745-754.

Martini A, Ciani M, and Scorzetti G (1996) Direct enumeration and isolation of wine yeasts from grape surfaces. Am J Enol Vitic 47(4): 435-440.

Nielsen JC and Richelieu M (1999) Control of flavor development in wine during and after malolactic fermentation by Oenococcus oeni. Appl environ microbiol 65(2): 740-745.

Ono B, Ishii N, Fujino S and Aoyama I (1991) Role of hydrosulfide ions (HS-) in methylmercury resistance in Saccharomyces cerevisiae. Appl Environ Microbiol 57(11): 3183-3186.

Pretorius IS (2000) Tailoring wine yeast for the new millennium: novel approaches to the ancient art of winemaking Yeast 16(8): 675-729.

Rainieri S and Pretorius I (2000) Selection and improvement of wine yeasts. Ann Microbiol 50(1): 15-32.
Romano P, Caruso M, Capece A, Lipani G, Paraggio $M$ and Fiore C (2003) Metabolic diversity of Saccharomyces cerevisiae strains from spontaneously fermented grape musts. World J Microbiol Biotechnol 19(3): 311-315.

Romano P, Capece A, Serafino V, Romaniello R and Poeta C (2008) Biodiversity of wild strains of Saccharomyces cerevisiae as a tool to complement and optimize wine quality. World J Microbiol Biotechnol 24(9): 1797-1802.

Shrestha B (2002) Practical biochemistry and biotechnology, 1st edition, pp 120-131

Spencer JFT and Spencer DM (2013) Yeasts in Natural and Artificial Habitats: Springer Berlin Heidelberg.

Torija MJ, Beltran G, Novo M, Poblet M, Guillamon JM, Mas A, and Rozes N (2003) Effects of fermentation temperature and Saccharomyces species on the cell fatty acid composition and presence of volatile compounds in wine. Int J Food Microbiol 85(1): 127-136.

Ugliano M, Kolouchova R and Henschke PA (2011) Occurrence of hydrogen sulfide in wine and in fermentation: influence of yeast strain and supplementation of yeast available nitrogen. J Ind Microbiol Biotechnol 38: 423-429.

Umeh SO (2016) Isolation, Characterization and Identification of Yeast (Saccharomyces Cerevisiae) From Three Local Beverage Drinks. Int J Series Multidiscip Res 2(5): 44-55.

Wang QM, Liu WQ, Liti G, Wang SA and Bai FY (2012) Surprisingly diverged populations of Saccharomyces cerevisiae in natural environments remote from human activity. Mol ecol 21(22): 5404-5417.

Wayman M and Parekh S (1990) Microbiology of fermentation catalysts. Biotechnology of Biomass Conversion. Milton Keynes: Open university press: $75-100$. 